\title{
BMJ Open Producing a preference-based quality of life measure for people with Duchenne muscular dystrophy: a mixed-methods study protocol
}

\author{
Philip A Powell, ${ }^{\oplus 1,2}$ Jill Carlton, ${ }^{1}$ Donna Rowen, ${ }^{1}$ John E Brazier ${ }^{1}$
}

To cite: Powell PA, Carlton J, Rowen $\mathrm{D}$, et al. Producing a preference-based quality of life measure for people with Duchenne muscular dystrophy: a mixed-methods study protocol. BMJ Open 2019;9:e023685. doi:10.1136/ bmjopen-2018-023685

- Prepublication history for this paper is available online. To view these files, please visit the journal online (http://dx.doi org/10.1136/bmjopen-2018023685).

Received 18 April 2018 Revised 6 December 2018 Accepted 28 January 2019

Check for updates

(c) Author(s) (or their employer(s)) 2019. Re-use permitted under CC BY-NC. No commercial re-use. See rights and permissions. Published by BMJ.

${ }^{1}$ School of Health and Related Research, University of Sheffield, Sheffield, UK

${ }^{2}$ Economics, University of Sheffield, Sheffield, UK

Correspondence to

Dr Philip A Powell;

p.a.powell@sheffield.ac.uk

\section{ABSTRACT}

Introduction Preference-based measures (PBMs) of health-related quality of life (HRQoL) are used to generate quality-adjusted life years, which are necessary for costeffectiveness evaluations of health interventions via costutility analysis. These measures of health can be generic (ie, pandiagnostic) or condition specific. No conditionspecific PBM of HRQoL in Duchenne muscular dystrophy (DMD) exists, yet there are concerns that standard generic measures lack the specificity to assess aspects of HRQOL that are especially important to people with DMD. This study has been designed to produce a condition-specific PBM of HRQOL in DMD.

Methods and analysis This mixed-methods study proceeds through three stages. In the first stage (concept elicitation), semistructured interviews will be conducted with boys and men diagnosed with DMD, and analysed with framework to produce a draft health state descriptive system for HRQoL in DMD. In the second stage (refining the descriptive system), patients, clinicians and primary caregivers of people with DMD will assess the face validity of the descriptive system. This will be followed by a quantitative survey on a larger sample of patients, which will be analysed with psychometric analyses to produce a refined descriptive system. In the third stage (valuation and econometric modelling), an online discrete choice experiment with duration will be administered to a general public sample to generate utility values for the new measure.

Ethics and dissemination This study has received ethica approval from the National Health Service (REC reference: 18/SW/0055). The primary output of this research will be a condition-specific PBM (or 'bolt-on' to an existing generic PBM) in people with DMD and an associated value set. Results will be disseminated through international conferences and open-access journals.

\section{INTRODUCTION}

Duchenne muscular dystrophy (DMD) is an inherited neuromuscular disorder that predominantly affects boys and men. It has an estimated incidence of 1:3800 to 1:6300 in live births. ${ }^{1}$ The disease causes progressive muscle weakness due to an absence of the dystrophin protein, which functions to help

\section{Strengths and limitations of this study}

- This study will produce a systematic health state descriptive system for describing health-related quality of life (HRQLL) in people with Duchenne muscular dystrophy (DMD) using complementary mixed methods.

- The primary output of the study will be the first condition-specific preference-based measure of $\mathrm{HRQOL}$ in people with DMD, which can be used in cost-effectiveness evaluations of new interventions.

- The study features input from a multidisciplinary team working with the charity Duchenne UK, clinicians, patients and primary caregivers of people with DMD.

- Given the recruitment methods, there is likely to be some selection bias in the samples, and sample sizes in some parts of the study (quantitative patient surveys) will be acceptable but may be lower than optimal due to DMD being a rare disease.

- The design and validity of the measure and accompanying value set are restricted to the UK, and will not be demonstrated internationally without further study.

keep muscle cells intact. Diagnostic symptoms and functional impairment are evident from as early as 2 years old and average life expectancy of people with DMD is approximately 25 years, ${ }^{2}$ although increasingly people with DMD are surviving into their fourth and even fifth decades. ${ }^{34}$ The disease progresses through four recognised clinical stages characterised by increased muscle weakness, impaired ambulation and motor functioning, and cardiovascular and respiratory problems. ${ }^{5}$ There is no known cure for the disease. Current clinical efforts are thus focused on improving the health-related quality of life (HRQoL) of people with DMD, which is considerably lower than that expected in a state of perfect health. ${ }^{4}$ Health interventions, therefore, are necessarily evaluated for their cost-effectiveness in improving HRQoL. The 
most common form of economic evaluation of health interventions is cost-utility analysis, which is used to compare interventions based on their cost per quality-adjusted life year (QALY). ${ }^{6}$ The QALY is a single measure that combines information on changes in both quantity of life and HRQoL, and is required by the National Institute for Health and Care Excellence (NICE) in the UK during health technology assessment. ${ }^{7}$ In order to derive a QALY, utilities (or preference weights) for different health states are required, which are often obtained from generic preference-based measures (PBMs) of health. PBMs of health have two components: a health state descriptive system that can be used to describe all possible different health states defined by a set of multilevel dimensions; and a set of utility weights (or value set) that represent the relative preferences people have for the different health states. Development of a PBM, thus, typically involves a mixedmethods approach: involving qualitative and subsequent quantitative psychometric methods for the generation and validation of the descriptive system, and a valuation survey for the value set. ${ }^{6}$

PBMs can either be generic, and designed for use across all health conditions, or condition specific. Previous PBMs used to assess HRQoL in DMD have been generic and have included the EuroQol-5-Dimensions (EQ-5D) ${ }^{89}$ and the Health Utilities Index (HUI) ${ }^{10}$ Generic measures are often recommended in the assessment of health technologies, as they allow for relative comparisons across health conditions. However, generic measures often lack the specificity to assess aspects of HRQoL that may be especially important to a particular health condition or group of health conditions. For example, neither the EQ-5D nor the HUI appears to adequately measure fatigue,${ }^{11-14}$ social participation ${ }^{12}{ }^{15-17}$ or dignity, ${ }^{14} 1819$ which have been shown to be important aspects of HRQoL for people with DMD. The HUI has a greater number of dimensions (and thus greater coverage) than the EQ-5D, and some limited research suggests the HUI may outperform the EQ-5D in people with disabilities, such as on proxy reliability. ${ }^{20}$ However, the demonstration of adequate measurement properties of these generic PBMs in people with neurodisability is lacking. ${ }^{21}$ Neither of these generic PBMs cover the full range of aspects of HRQoL that may be important to people with muscular dystrophies, including DMD. ${ }^{22}$ This is problematic, as they have been used to generate HRQoL utilities for economic evaluations in DMD. ${ }^{23}$ Quality of life measures that have been designed for use in people with DMD, such as the Quality of Life in Neuromuscular Disease instrument, ${ }^{24}$ are not preference based and thus cannot be used to generate QALYs directly. Moreover, different measures may be used across different clinical stages of DMD and throughout the life course, making it difficult to compare estimates of HRQoL on a common index, which is desirable for users to inform health technology assessment.

This study has been designed to evaluate the content validity of three existing, well-used generic PBMs of HRQoL (the EQ-5D for adults or the EQ-5D-Y which is the youth version; the HUI, based on the HUI2 and HUI3 classification systems; and the Child Health Utility 9D [CHU-9D]), while generating the content for a new condition-specific PBM, or condition-specific 'bolt-on' addition to an existing PBM, calibrated for assessing HRQoL in people with DMD. Bolt-ons are additional dimensions added onto an existing measure where there is a concern that the measure does not capture all important dimensions, but where the existing dimensions including severity levels are appropriate. ${ }^{25-27}$ As is typical in studies that involve the development of patient-reported outcome measures, ${ }^{28}$ this research involves mixed methods and proceeds through three sequential stages.

In the first stage of concept elicitation, semistructured qualitative interviews will be conducted with boys and men diagnosed with DMD and analysed to produce a draft health state descriptive system for HRQoL in DMD. In the second stage of refining the descriptive system, the face validity and the psychometric properties of a questionnaire derived from the draft descriptive system will be assessed and improved using mixed methods, in order to produce a refined descriptive system and questionnaire for measuring HRQoL in DMD. In the third and final stage of valuation and econometric modelling, a discrete choice experiment with duration $\left(\mathrm{DCE}_{\mathrm{TTO}}\right)$ will be used in a survey of the general public, where they will be asked to value health states selected from the refined health state descriptive system in order to derive the value set. This protocol outlines the design and methodology, we will use in each of the three stages of the research (summarised in figure 1). The product will be a rigorous PBM or bolt-on(s) to an existing PBM, of HRQoL in people with DMD for use in the economic evaluation of health interventions.

This work is funded by the charity Duchenne UK and falls under the umbrella of 'Project Hercules' (Health Research Collaboration United in Leading Evidence Synthesis) led by the charity. It is designed to develop common tools and practices in health technology assessment for DMD to improve engagement between industry and international agencies such as NICE on decisions on which medicines and treatments to fund. This includes a need for a robust and valid preference-based HRQoL metric to use to assess the cost-effectiveness of treatments.

\section{AIMS}

This study has the following three aims:

1. Using both bottom-up (qualitative) and top-down (review) methods generates a draft descriptive system for measuring HRQoL in people with DMD.

2. Using face validity and psychometric analyses with patients with DMD, and their primary carers and clinicians refine the initial draft descriptive system generated in (1) to produce a final, refined descriptive system, suitable for assessing HRQoL in DMD.

3. Design and conduct a valuation study using $\mathrm{DCE}_{\text {TTO }}$, with the adult UK general public to produce utility 


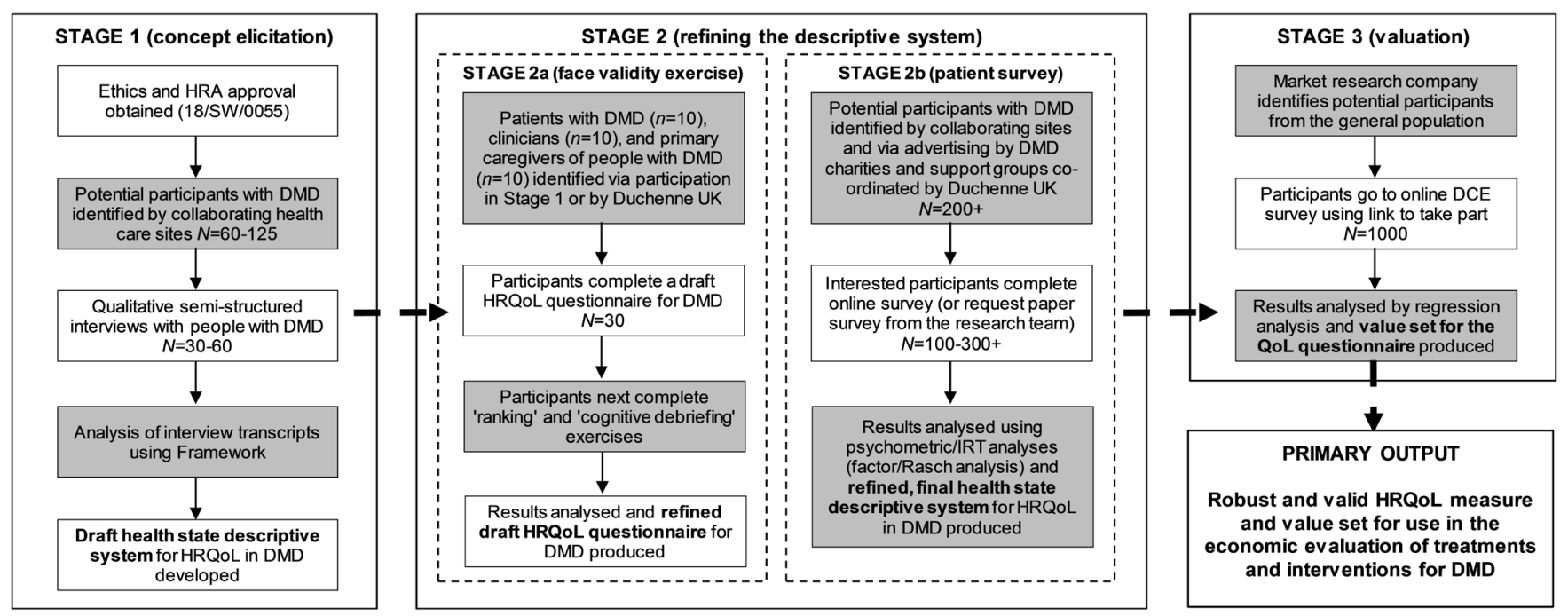

Figure 1 Research project process diagram. Design stages omitted. DCE, discrete choice experiment; DMD, Duchenne muscular dystrophy; HRA, Health Research Authority; HRQoL, health-related quality of life; IRT, Item response theory; QoL, quality of life.

values for all health states defined by the descriptive system generated in (2).

\section{METHODS AND ANALYSIS}

\section{Stage 1: concept elicitation}

\section{Study design and procedure}

\section{Development of a topic guide}

A topic guide or 'interview schedule' will be used to help provide structure to the semistructured qualitative interviews and ensure that all important a priori information on HRQoL in people with DMD is covered. ${ }^{29}$ The topic guide will be informed by a rapid scoping review of the literature on HRQoL themes in DMD,${ }^{30}$ and the content of the generic PBMs of HRQoL assessed in this study (ie, the EQ-5D-Y/EQ-5D, HUI and CHU-9D). Experts through experience, including clinicians and caregivers to people with DMD, will be asked to informally review the topic guide to evaluate its face validity and inclusivity, prior to the interviews. These experts will be identified by Duchenne UK, as part of Project Hercules advisory groups. The selection of experts informing this project will be intended to generate a breadth of clinical expertise, but will ultimately be determined by convenience sampling (ie, based on experts' availability in accordance with the project timelines).

\section{Qualitative interviews}

Participants with a primary diagnosis of DMD will be sent an invitation to take part in a $60 \mathrm{~min}$ semistructured interview exploring the domains of their HRQoL. The semistructured topic guide ensures important topics of interest are covered, while allowing the flexibility for elaboration and follow-ups in the case of interesting or ambiguous points made by the interviewee. ${ }^{29}$ Across a number of areas, participants will be asked about their HRQoL and whether the EQ-5D-Y/EQ-5D, HUI or CHU-9D adequately captures this dimension. Participants will also be asked to provide details on any dimensions of HRQoL that are important to them but are not covered in the topic guide.

Participants will be sent a study pack directly by post from a recruiting National Health Service (NHS) site (see below), but will have to contact the research team should they wish to take part. The study pack will contain an invitation letter, information sheet, consent form, background details form and interview schedule. This content allows the participant to familiarise themselves with the material, and thus maximise the quality of subsequent interview data and informed consent of what is involved. ${ }^{31}$ Participants will also be given a template notification letter for their general practitioner (GP), which they can pass on if and when they decide to disclose their participation in the study to their GP.

One experienced qualitative researcher will conduct the interviews to ensure consistency throughout. To facilitate inclusivity, interviews will take place in the preferred location/medium and time for the participant. Interviews could thus take place face to face on NHS sites, University premises, participants' homes and remotely via the telephone and Skype, where necessary (tools like Skype have been shown to be a valid alternative to face-to-face interviews in qualitative methods) ${ }^{32}$ While it is preferred that the interviews take place with the participants alone (to avoid biased responses), the presence of others, such as a caregiver, and/or parent(s) and guardian(s) in the case of children (under 16 years old), will be facilitated on the participant's and/or consenting parent's wishes. In all cases with children, the parent or guardian will be asked to remain in the vicinity of the interview (ie, in the same building). Participants or a parent/guardian in the case of children will give their informed consent prior to data collection, using a consent form. All interviews 
will be digitally recorded on an encrypted device, transcribed verbatim by the research team and anonymised. As well as taking part in an interview, participants will complete a brief background details form, indicating some broad clinical and background characteristics to ensure we interview a sufficiently broad cross-section of people with DMD. These background characteristics include brief questions on lower and upper limb mobility, ventilator use, use of steroid and heart medication, and age. All documents and the study methodology have been approved by the Duchenne UK Project Hercules advisory groups.

\section{Producing a draft descriptive system}

The qualitative data will be analysed to produce themes, used to populate a draft health state descriptive system of HRQoL in DMD. The draft descriptive system will be used to generate multiple HRQoL items as part of a questionnaire to be administered in a patient sample of people with DMD in stage 2. The draft descriptive system will be circulated for expert comments and feedback from Duchenne UK via the Project Hercules advisory groups. As well as from participants themselves, by 'member checking', the results to make sure they are consistent with participants' perspectives. ${ }^{33}$ This methodology has been previously applied by members of the research team to generate descriptive systems for PBMs in other health conditions. ${ }^{345}$ Assuming, as predicted, that generic PBMs (ie, the EQ-5D-Y/EQ-5D, HUI and CHU-9D) are insufficient to capture all important domains uncovered by the qualitative research, the research project will progress onto stage 2. If, in the unlikely event that, the generic PBMs capture all important domains of HRQoL to people with DMD, then we would not continue with the latter stages of the research, and would instead inform all relevant parties, including the relevant research ethics committee and transparently report on our findings as they have emerged.

\section{Study sample and recruitment}

Participants for stage 1 will be identified by NHS healthcare teams across five collaborating sites in the UK that specialise in the care and treatment of children and/ or adults with DMD. The sites will identify and contact potential participants directly with a study pack (without any disclosure of patient data to the research team). Participants will then need to contact the research team if they are interested in taking part. Potential participants will be purposively sampled, in order to ensure a sufficient degree of representation across age, lower and upper limb function, and respiratory function, as advised by families of people with DMD via Duchenne UK. An illustrative sampling framework is shown in table 1 . Recruitment will proceed in an iterative fashion, whereby these selection characteristics are monitored to ensure a good balance across them. Participants will be recruited to ensure coverage across the life course of people with DMD, from childhood to adulthood. A minimum age of 7
Table 1 An example sampling framework for a recruiting site, based on a maximum identified sample of 25 potential participants

\begin{tabular}{|c|c|c|c|c|c|c|c|c|}
\hline \multicolumn{9}{|l|}{ Clinical criteria } \\
\hline \multicolumn{9}{|l|}{$\begin{array}{l}\text { Lower limb } \\
\text { function }\end{array}$} \\
\hline $\begin{array}{l}\text { Gross upper limb } \\
\text { function }\end{array}$ & $\checkmark$ & $\checkmark$ & \multicolumn{2}{|r|}{$\checkmark$} & \multicolumn{2}{|r|}{$x$} & \multicolumn{2}{|c|}{$x$} \\
\hline $\begin{array}{l}\text { Respiratory } \\
\text { function }\end{array}$ & $\checkmark$ & $\checkmark$ & \multicolumn{2}{|c|}{$x$} & \multicolumn{2}{|r|}{$\checkmark$} & \multicolumn{2}{|c|}{$x$} \\
\hline \multicolumn{8}{|l|}{ Age group } & Total \\
\hline \multicolumn{3}{|c|}{ Preadolescence (<10years) } & 2 & 2 & 0 & 0 & 0 & 4 \\
\hline \multicolumn{3}{|c|}{ Early adolescence (10-14 years) } & 2 & 2 & 1 & 1 & 0 & 6 \\
\hline \multicolumn{3}{|c|}{ Late adolescence (15-19years) } & 0 & 2 & 2 & 2 & 1 & 7 \\
\hline \multicolumn{3}{|c|}{ Early adulthood (20-29years) } & 0 & 0 & 1 & 2 & 2 & 5 \\
\hline \multicolumn{3}{|c|}{ Middle adulthood (30-39years) } & 0 & 0 & 0 & 0 & 2 & 2 \\
\hline \multicolumn{3}{|c|}{ Late adulthood (>39years) } & 0 & 0 & 0 & 0 & 1 & 1 \\
\hline
\end{tabular}

'Lower limb function' is based on the ability to walk independently without a mobility device; 'gross upper limb function' is based on the ability to raise a hand to mouth to eat/drink independently; 'respiratory function' is based on the absence of any ventilation. Cell counts represent crosstabulations between clinical criteria and age group, and are approximations.

years has been applied, based on previous work suggesting children at this age are capable of reporting on aspects of their HRQoL. ${ }^{36}$ The inclusion and exclusion criteria for each stage of the study are illustrated in table 2.

The sample size for the qualitative research interviews will be determined by data saturation. Data saturation is achieved when no additional novel themes are emerging from interviews with participants and a sufficient number of participants with different characteristics (as defined above) have been interviewed. While it is impossible to determine the exact number of participants required to reach data saturation in advance, prior studies of patients' perspectives on HRQoL in different specific health conditions provide an informed estimate of between 30 and 60 people. ${ }^{345}$ Assuming a 50\% uptake rate from potential participants, each site will be asked to identify between 12 and 25 potential participants.

\section{Data analysis plan}

The interview transcripts in stage 1 will be subjected to thematic content analysis using framework, an approach developed in the context of applied policy research. ${ }^{37}$ Briefly, framework is a form of thematic content analysis that involves coding and identifying common categories emerging from the data, which are indexed and then grouped into themes (or attributes). NVivo qualitative analysis software will be used to facilitate and manage the analysis. The trustworthiness (quality) of the qualitative analysis will be assured using the four criteria of trustworthiness, which includes 'member checking'. ${ }^{33}$ Stage 1 of the project is estimated to take approximately 12 months. 
Table 2 Sample inclusion/exclusion criteria for the three study stages Inclusion criteria

\section{Exclusion criteria}

\begin{tabular}{|c|c|c|}
\hline \multirow[t]{3}{*}{ Stage 1} & Boys and men diagnosed with DMD & $\begin{array}{l}\text { Women with DMD or those with other forms of muscular } \\
\text { dystrophy }\end{array}$ \\
\hline & Aged $7+$ years old & Aged $<7$ years old \\
\hline & $\begin{array}{l}\text { Have the capacity to consent (or receive parental } \\
\text { consent if }<16 \text { years old) }\end{array}$ & $\begin{array}{l}\text { Lacking in the capacity to consent (or receive parental } \\
\text { consent) }\end{array}$ \\
\hline \multirow[t]{2}{*}{ Stage $2 b$} & Same criteria as stage 1 for patients & Same criteria as stage 1 for patients \\
\hline & $\begin{array}{l}\text { Proxy or assisted reporting by people aged } \\
18+\text { years old who have the appropriate authority to } \\
\text { respond on behalf of a patient diagnosed with DMD, } \\
\text { fluent in English, possessing the capacity to consent. }\end{array}$ & $\begin{array}{l}\text { People who are not providing proxy or assisted } \\
\text { reporting for, or do not have the appropriate authority to } \\
\text { respond on behalf of, patients diagnosed with DMD, or } \\
\text { those who do who do not meet the inclusion criteria }\end{array}$ \\
\hline & Aged $18+$ years old & Aged $<18$ years old \\
\hline & Fluent in English & Unable to understand or speak English \\
\hline & Have the capacity to consent & Lacking in the capacity to consent \\
\hline
\end{tabular}

Additional iterative sampling strata apply to the qualitative interviews to ensure sufficient breadth of coverage.

DMD, Duchenne muscular dystrophy.

\section{Stage 2: refining the descriptive system}

Study design and procedure

Stage 2a: ensuring face validity

In stage $2 \mathrm{a}$, a small sample of patients with $\mathrm{DMD}$, primary caregivers of people with DMD and clinicians will be invited to assess the face validity of the draft descriptive system. They will be asked to complete a series of tasks to check that the questionnaire is suitable for administration in a larger sample (ie, that it can be understood by all parties and makes sense, including the wording of instructions, items and response options). This exercise will be completed in the presence of the researcher, and, to minimise participant burden, will be offered face to face or remotely (eg, via Skype or telephone) as in the interviews for stage 1. Participants (which includes patients who have taken part in stage 1; see below) will be sent an information sheet and consent form in advance to give them time to decide whether or not to take part in the study. Participants will provide consent in the same way as that in stage 1 (above), and the same procedural details will apply.

Following consent, participants will be asked to complete the draft questionnaire in the presence of the researcher (either physically or remotely) to check that they can fill it in accurately and understand it. Following this, participants will be asked to complete a ranking exercise to determine the ordering of the response levels for the questionnaire, and a 'cognitive debriefing' exercise (where participants are asked what they think the items mean) to ensure that the items are measuring what they are intended to. If any patients have not provided background details as part of an interview in stage 1, this will also be included. In place of basic aggregate clinical background details, we will collect basic age (decade ranges) and gender information from clinicians and primary caregivers of people with DMD to provide basic descriptive background on the sample. Participants will also be given a template notification letter for their GP which they can pass on if and when they decide to disclose their participation in the study to their GP. These methods have previously been used by members of the research team in developing condition-specific measures in other health conditions. ${ }^{35} 38$ This exercise will not be recorded, but anonymous notes will be taken by the researcher on potential modifications to the questionnaire to make it as fit-for-purpose and user-friendly as possible. In total, the face validity exercise is expected to take approximately $30 \mathrm{~min}$.

\section{Stage $2 b$ : quantitative surveying}

In stage $2 b$, quality of life items derived from the descriptive system developed in stage 1 (refined as necessary in stage 2a), and a selection of generic PBMs informed by the results of stage 1 to facilitate comparability (eg, the EQ-5D-Y/EQ-5D, HUI and/or the CHU-9D), will be administered to as wide as possible a patient sample of people with DMD. Participants with a diagnosis of DMD will be invited to complete a survey online, or offline by 
request to the researchers for a paper copy. Potential participants will be sent a study pack directly by post from a recruiting NHS site (see below) or respond to adverts circulated by charities and support groups, coordinated by Duchenne UK. Participants will be required to read an information sheet and provide their informed consent (or the consent of a parent or guardian in the case of children) prior to completing the survey. These documents will be enclosed with the invitation letter for participants and made compulsory reading on the survey website prior to accessing the study measures. While self-reported responses are desirable, to facilitate inclusivity we will make the survey as accessible as possible to assisted self-report and proxy responders. Participants will be asked to note on the survey whether they are completing it themselves (self-report), with help from another (assisted self-report), or someone is completing it on their behalf (proxy). It is anticipated that the survey will take up to 10 min of participants' time.

\section{Producing a refined health state descriptive system}

The quantitative data from the surveys of people with DMD in stage $2 \mathrm{~b}$ will be subjected to psychometric analyses (see below), along with expert input from the Project Hercules advisory groups, to produce a final health state descriptive system. ${ }^{39}$ Depending on the results of stage 1 , stage 2 and expert input, this will either form a new HRQoL measure, or 'bolt-on' dimensions to an existing generic PBM, such as the EQ-5D-Y/EQ-5D, HUI or CHU-9D.

\section{Study sample and recruitment}

In stage 2a (face validity exercise), participants will be those patients that have taken part in stage 1 and have consented to be contacted for further research by the research team, and clinicians and primary caregivers of people with DMD identified by Duchenne UK via their advisory groups. This is a pilot activity designed to ensure the questionnaire is 'fit-for-purpose' prior to administration in a larger sample. A total sample size of 30 participants, composed of 10 clinicians, 10 primary caregivers of and 10 people with, DMD, based on similar previous face validity work. ${ }^{38}$ In the event that more than 10 people with DMD from stage 1 have agreed to be contacted for further research, participants will be selected to ensure breadth across the stage 1 sampling framework in (table 1).

In stage $2 b$ (quantitative patient survey), participants will be those that have taken part in stage 1 and have consented to be contacted for further research by the research team; additional participants identified by the healthcare team; or additional participants identified via advertisements circulated by DMD charities and support groups (coordinated by Duchenne UK). The identification of potential participants by the healthcare team will proceed in the same fashion as that in stage 1 (with study packs provided to potential participants in their care). The number which they identify will be as many as possible that meet the inclusion or exclusion criteria, but this will be preagreed with sites to align with capacity (with an optimal number of approximately 40 additional participants per site). While similar prior studies have used samples of approximately 342-655 patients, ${ }^{39} 40$ as DMD is a rare health problem, it is important to recognise that such numbers may be difficult to achieve in practice. Recent simulations have suggested that sample sizes of at least 100 people and above produce stable estimates in Rasch analyses, ${ }^{41}$ from which to draw conclusions, and so we have set this as our target minimum sample size, while aiming for an optimal sample of 300 people or greater.

\section{Data analysis plan}

In stage 2a, face validity will be assessed using ranking and debriefing exercises in a small sample of DMD patients, primary caregivers and clinicians. This will also involve taking anonymous notes of the responses and making refinements to the draft questionnaire as a result of feedback. Similar methods have been used to help develop condition-specific measures for amblyopia and self-management in diabetes. ${ }^{35}{ }^{38}$ Refinement of the descriptive system in stage $2 \mathrm{~b}$ will involve factor analysis, Rasch analysis and standard psychometric analyses as used in previous work by members of this group, with the aim of producing a health state descriptive system appropriate for measuring the HRQoL of people with DMD. ${ }^{39}{ }^{40}$ Factor analysis will be used to examine domains. Rasch and standard psychometric analyses will be used to examine differential item functioning across important subgroups (where feasible given the sample size), item response distributions, item severity, ability for items to discriminate by severity, whether item response options are ordered by severity and correlations between items. In the assessment of items, these analyses will be combined based on prior predefined criteria used in previous measure development by this research group. ${ }^{42}$ Alongside all previous results and analyses in the study, this psychometric analysis will be used to ensure the best performing items remain in the final measure used for valuation in stage 3. Stage 2 of the project is estimated to take approximately 4 months.

\section{Stage 3: valuation and econometric modelling Study design and procedure Designing the DCE}

An increasingly widely used preference elicitation technique for valuing health is the $\mathrm{DCE}_{\text {тто }}$, which is a technique that can be conducted online and data can be modelled to generate utility values for all states defined by the descriptive system. ${ }^{43} 44$ The technique asks respondents to choose between two profiles, where each profile is described using a severity level of each of the dimensions from the descriptive system, plus a duration level (based on remaining life expectancy). Respondents are asked to choose which profile they think is best. The duration attribute will be 1, 4, 7 and 10 years, as used in recent surveys. ${ }^{445}$ Profiles will be selected for inclusion in the $\mathrm{DCE}_{\text {тто }}$ using a D-Optimal design using 
the specialist software Ngene. Illogical combinations of the attributes will be identified and excluded from the design. The design will also select which profile appears as $\mathrm{A}$ or $\mathrm{B}$, and the combinations of profiles that each respondent values. Based on the expected size of the descriptive system, 200 health profile combinations will be valued, and each respondent will undertake 10 tasks, leading to 50 observations per health profile combination. In the $\mathrm{DCE}_{\text {Tто }}$, respondents will be asked to imagine the hypothetical health profiles for themselves, and asked which they think is best. They will not be told the underlying health condition (DMD) or that the profile is typically experienced by children, which causes problems regarding people's willingness to sacrifice a reduction in life expectancy to improve the health of the child (which is the method the technique uses to generate utility values). This perspective has been taken in other child valuation surveys, including the UK valuation of the CHU-9D, ${ }^{46}$ and the Netherlands valuation of the CHU-9D, which also used the DCE with duration technique conducted online. ${ }^{45}$

\section{Conducting the DCE}

In order to produce utility values for health states defined by the health state descriptive system, $\mathrm{DCE}_{\text {Tто }}$ will be used in an online survey of 1000 members of the UK adult general population. The adult general population was also used in the UK and Netherlands valuation of the CHU-9D, and is consistent with the NICE methods guide for valuation, where adult general population values are sought rather than patient values. ${ }^{7}$ Furthermore, the elicitation of child values presents a large number of challenges around whether they are cognitively able to undertake health state valuation tasks and little research has been undertaken on this. ${ }^{47}$ The survey will be hosted on Qualtrics, survey software supported at the host research institution. Participants will read an information sheet online and provide their informed consent online at the start of the survey. The survey will have three parts. First participants will complete questions about themselves and their health, second they will complete one practice question and $10 \mathrm{DCE}_{\text {тто }}$ questions, and finally, they will report what they thought of the survey. Participants successfully completing the survey will receive a predetermined reward from the market research agency in line with the agencies usual procedure.

\section{Valuation using the DCE}

Health states will be valued using the $\mathrm{DCE}_{\text {тто }}$ data derived from the online survey using standard regression techniques (see below). This exercise will produce utility values for each (health state) attribute level defined by the condition-specific PBM of HRQoL in DMD, for use in cost-effectiveness evaluations of new interventions.

\section{Study sample and recruitment}

Identification and recruitment of the sample for stage 3 will be outsourced to a reputable market research company that conforms to governance standards (eg, the Market Research Society Code of Conduct). Participants will be invited to take part in the survey through email or via a portal by the market research agency with a link to the online survey. This method of recruitment has been used successfully by members of the research team on previous health valuation projects. ${ }^{45} 48$ A predetermined sample size of 1000 people from the general population will be used. This sample size has been selected to ensure that each pair of health profiles is valued well in excess of a minimum of 20 times and a minimum of one pair of profiles per parameter estimated is selected in the regression model. ${ }^{49}$

\section{Data analysis plan}

The DCE $_{\text {тто }}$ surveys completed in stage 3 will be analysed by regression analysis to produce utility values for health states defined by the health state descriptive system. ${ }^{43}$ The utility value of each level of each attribute is generated using the 'marginal rate of substitution', generated by dividing the coefficient for each level of each attribute by the coefficient for duration, producing the utility value for each (health state) attribute level. Choices will be modelled using the conditional logistic model. Sensitivity analysis will be undertaken to assess the robustness of the results by excluding respondents. Similar methods have been used elsewhere by this group. ${ }^{45} 48$ Stage 3 of the project is estimated to take approximately 4 months.

\section{Patient and public involvement}

This research was driven by a recognised need within the Duchenne community to better measure quality of life in people with DMD. At the outset, their views were represented by the charity Duchenne UK, which includes mothers of sons with DMD. Duchenne UK, under the work stream of Project Hercules, has contributed to the design of the proposed project. There is an established patient and public involvement (PPI) group, which includes adults with DMD and parents of children with DMD. The group will contribute as research peers at several key stages of the project, including stage 1 and stage 2 during item generation and item selection. The research findings will be disseminated to participants via Duchenne UK, through established communication channels with members of the Duchenne community and a bespoke Project Hercules event scheduled for November 2019.

\section{ETHICS AND DISSEMINATION}

The work will be conducted in accordance with the ethical principles underlying the Declaration of Helsinki and good practice guidelines on the proper conduct of research. A full data management plan is in place to ensure the appropriate handling and use of personal and non-identifiable data, necessary for the successful conduct of this research. Advisory groups, in the form of steering 
and PPI groups, are being set up by Duchenne UK as part of Project Hercules to help advise and oversee the findings that emerge from the research, including advising on ethical and practical issues such as patient burden. All study documents and the study methodology have been approved by Duchenne UK and parents of patients with DMD.

The primary output of this research will be a condition-specific PBM (or bolt-on to an existing measure) for assessing HRQoL in people with DMD, and an associated value set for use in cost-effectiveness evaluations. The results of this project will be disseminated via at least two international conferences and two journal manuscripts (ie, one for stages $1 / 2$ and one for stage $3)$. The manuscripts will be published open access to ensure the findings of the research are freely available, transparent and accessible to all with an interest in reading about them. Non-technical report(s) of the findings will also be circulated to study stakeholders via Duchenne UK.

Acknowledgements We would like to thank Ros Quinlivan, Natalie Wilson, Julie Woodley and members of the NHS South West-Central Bristol Research Ethics Committee, our collaborating health care sites, Duchenne UK, patient advisers and the Project Hercules advisory groups for their ongoing input, feedback and contributions into the design of this study.

Contributors JC, DR and JEB conceived the study. PAP, JC and DR contributed to the design of the study. PAP wrote the manuscript. All authors contributed to and approved the manuscript.

Funding This work was supported by Duchenne UK under the Project Hercules funding stream.

Competing interests None declared.

Patient consent for publication Not required.

Ethics approval This study has been ethically reviewed and received Health Research Authority approval and a favourable ethical opinion from the NHS South West-Central Bristol Research Ethics Committee (REC reference: 18/SW/0055) on 14 March 2018.

Provenance and peer review Not commissioned; externally peer reviewed.

Open access This is an open access article distributed in accordance with the Creative Commons Attribution Non Commercial (CC BY-NC 4.0) license, which permits others to distribute, remix, adapt, build upon this work non-commercially, and license their derivative works on different terms, provided the original work is properly cited, appropriate credit is given, any changes made indicated, and the use is non-commercial. See: http://creativecommons.org/licenses/by-nc/4.0/.

\section{REFERENCES}

1. Mendell JR, Shilling C, Leslie ND, et al. Evidence-based path to newborn screening for Duchenne muscular dystrophy. Ann Neurol 2012;71:304-13.

2. Strehle EM, Straub V. Recent advances in the management of Duchenne muscular dystrophy. Arch Dis Child 2015;100:1173-7.

3. Kieny P, Chollet S, Delalande P, et al. Evolution of life expectancy of patients with Duchenne muscular dystrophy at AFM Yolaine de Kepper centre between 1981 and 2011. Ann Phys Rehabil Med 2013;56:443-54.

4. Ryder S, Leadley RM, Armstrong N, et al. The burden, epidemiology, costs and treatment for Duchenne muscular dystrophy: an evidence review. Orphanet J Rare Dis 2017;12:79.

5. Landfeldt E, Lindgren P, Bell CF, et al. Compliance to care guidelines for duchenne muscular dystrophy. J Neuromuscul Dis 2015;2:63-72.

6. Brazier J, Ratcliffe J, Salomon JA, et al. Measuring and valuing health benefits for economic evaluation. Oxford, UK: Oxford University Press, 2017.
7. NICE (National Institute for Health and Care Excellence). Guide to the methods of technology appraisal. London, UK: NICE, 2013.

8. Cavazza M, Kodra Y, Armeni P, et al. Social/economic costs and health-related quality of life in patients with Duchenne muscular dystrophy in Europe. Eur J Health Econ 2016;17(Suppl 1):19-29.

9. Landfeldt E, Lindgren P, Bell CF, et al. Quantifying the burden of caregiving in Duchenne muscular dystrophy. J Neurol 2016;263:906-15.

10. Landfeldt E, Lindgren P, Bell CF, et al. Health-related quality of life in patients with Duchenne muscular dystrophy: a multinational, crosssectional study. Dev Med Child Neurol 2016;58:508-15.

11. Houwen-van Opstal SL, Jansen M, van Alfen N, et al. Healthrelated quality of life and its relation to disease severity in boys with Duchenne muscular dystrophy: satisfied boys, worrying parents--a case-control study. J Child Neurol 2014;29:1486-95.

12. Pangalila RF, van den Bos GA, Bartels $B$, et al. Prevalence of fatigue pain, and affective disorders in adults with duchenne muscular dystrophy and their associations with quality of life. Arch Phys Med Rehabil 2015;96:1242-7.

13. Wei Y, Speechley KN, Zou G, et al. Factors Associated With Health-Related Quality of Life in Children With Duchenne Muscular Dystrophy. J Child Neurol 2016;31:879-86.

14. Wei S, Campbell C, Speechley K. Health-related quality of life in children with Duchenne Muscular Dystrophy. Can J Neurol Sci 2014;41:S15.

15. Bendixen RM, Senesac C, Lott DJ, et al. Participation and quality of life in children with Duchenne muscular dystrophy using the International Classification of Functioning, Disability, and Health. Health Qual Life Outcomes 2012;10:43.

16. Bendixen RM, Lott DJ, Senesac C, et al. Participation in daily life activities and its relationship to strength and functional measures in boys with Duchenne muscular dystrophy. Disabil Rehabil 2014;36:1918-23.

17. Heutinck L, Kampen NV, Jansen M, et al. Physical activity in boys with duchenne muscular dystrophy is lower and less demanding compared to healthy boys. J Child Neurol 2017;32:450-7.

18. Madsen A, Rahbek J, Werge B, et al. Living conditions and quality of life in adults with Duchenne muscular dystrophy-A Danish survey. Neuromuscul Disord 2014;24:913.

19. Martinsen B, Dreyer P. Dependence on care experienced by people living with Duchenne muscular dystrophy and spinal cord injury. J Neurosci Nurs 2012;44:82-90.

20. Bray N, Noyes J, Harris N, et al. Measuring the health-related quality of life of children with impaired mobility: examining correlation and agreement between children and parent proxies. BMC Res Notes 2017;10:377.

21. Janssens A, Rogers M, Gumm R, et al. Measurement properties of multidimensional patient-reported outcome measures in neurodisability: a systematic review of evaluation studies. Dev Med Child Neurol 2016;58:437-51.

22. Bann CM, Abresch RT, Biesecker B, et al. Measuring quality of life in muscular dystrophy. Neurology 2015;84:1034-42.

23. Landfeldt E, Alfredsson L, Straub V, et al. Economic evaluation in duchenne muscular dystrophy: model frameworks for costeffectiveness analysis. Pharmacoeconomics 2017;35:249-58.

24. Dany A, Barbe C, Rapin A, et al. Construction of a Quality of Life Questionnaire for slowly progressive neuromuscular disease. Qual Life Res 2015;24:2615-23.

25. Finch AP, Brazier JE, Mukuria C, et al. An exploratory study on using principal-component analysis and confirmatory factor analysis to identify bolt-on dimensions: the EQ-5D case study. Value Health 2017;20:1362-75.

26. Yang Y, Rowen D, Brazier J, et al. An exploratory study to test the impact on three "bolt-on" items to the EQ-5D. Value Health 2015;18:52-60.

27. Yang Y, Brazier J, Tsuchiya A. Effect of adding a sleep dimension to the EQ-5D descriptive system: a "bolt-on" experiment. Med Decis Making 2014;34:42-53.

28. Martin ML, Blum SI, Liedgens $\mathrm{H}$, et al. Mixed-methods development of a new patient-reported outcome instrument for chronic low back pain: Part 1 - The patient assessment for low back pain - symptoms (PAL-S). Pain 2018.

29. Stevens K, Palfreyman S. The use of qualitative methods in developing the descriptive systems of preference-based measures of health-related quality of life for use in economic evaluation. Value Health 2012;15:991-8.

30. Uttley L, Carlton J, Woods HB, et al. A review of quality of life themes in Duchenne muscular dystrophy for patients and carers. Health Qual Life Outcomes 2018;16:237. 
31. Jepson M, Abbott D, Hastie J. "This is another personal question": Research interviews and discussing sensitive issues with men with life-limiting conditions. Int J Mens Health 2015;14:273-86.

32. lacono VL, Symonds P, Brown DHK. Skype as a tool for qualitative research interviews. Sociol Res Online 2016;21:12.

33. Lincoln YS, Guba EG, Pilotta JJ. Naturalistic Inquiry. Newbury Park: Sage, 1985:438-9.

34. Carlton J. Identifying potential themes for the Child Amblyopia Treatment Questionnaire. Optom Vis Sci 2013;90:867-73.

35. Carlton J, Elliott J, Rowen D, et al. Developing a questionnaire to determine the impact of self-management in diabetes: giving people with diabetes a voice. Health Qual Life Outcomes 2017:15:146.

36. Stevens KJ. Working with children to develop dimensions for a preference-based, generic, pediatric, health-related quality-of-life measure. Qual Health Res 2010;20:340-51.

37. Ritchie J, Spencer L. Qualitative data analysis for applied policy research. In: Bryman BB, Burgess R, eds. Analyzing Qualitative Data. London: Routledge, 1994-.

38. Carlton J. Developing the draft descriptive system for the child amblyopia treatment questionnaire (CAT-Qol): a mixed methods study. Health Qual Life Outcomes 2013;11:174.

39. Carlon J. Refinement of the Child Amblyopia Treatment Questionnaire (CAT-QoL) using Rasch analysis. HEDS Discussion Paper No. 13.13. 2013. Available at https://www.sheffield.ac.uk/ polopoly fs/1.321886!/file/13.13.pdf.

40. Rowen D, Brazier J, Young T, et al. Deriving a preference-based measure for cancer using the EORTC QLQ-C30. Value Health 2011:14:721-31.

41. Chen WH, Lenderking W, Jin $Y$, et al. Is Rasch model analysis applicable in small sample size pilot studies for assessing item characteristics? An example using PROMIS pain behavior item bank data. Qual Life Res 2014;23:485-93.

42. Keetharuth AD, Taylor Buck E, Acquadro C, et al. Integrating Qualitative and Quantitative Data in the Development of Outcome Measures: The Case of the Recovering Quality of Life (ReQoL) Measures in Mental Health Populations. Int J Environ Res Public Health 2018;15:1342.

43. Bansback N, Brazier J, Tsuchiya A, et al. Using a discrete choice experiment to estimate health state utility values. $J$ Health Econ 2012;31:306-18.

44. Norman R, Viney R, Brazier J, et al. Valuing SF-6D Health States Using a Discrete Choice Experiment. Med Decis Making 2014:34:773-86.

45. Rowen D, Mulhern B, Stevens K, et al. Estimating a Dutch Value Set for the Pediatric Preference-Based CHU9D Using a Discrete Choice Experiment with Duration. Value Health 2018;21:1234-42.

46. Stevens K. Valuation of the Child Health Utility 9D Index. Pharmacoeconomics 2012;30:729-47.

47. Stevens K. "Because that's what matters to me". A pilot study to test the feasibility and reliability of ordinal valuation methods for health state valuation with children. HEDS Discussion Paper, University of Sheffield. 2015. available at https://www.sheffield.ac.uk/polopoly_fs/ 1.526959!/file/K.Stevens The feasibility of health state valuation by_children_DPfinal.pdf.

48. Rowen D, Stevens K, Labeit A, et al. Using a discrete-choice experiment involving cost to value a classification system measuring the quality-of-life impact of self-management for diabetes. Value Health 2018;21:69-77.

49. Lancsar E, Louviere J. Conducting discrete choice experiments to inform healthcare decision making: a user's guide. Pharmacoeconomics 2008;26:661-77. 